\title{
NONLOCAL PROBLEM FOR A MIXED TYPE FOURTH-ORDER DIFFERENTIAL EQUATION WITH HILFER FRACTIONAL OPERATOR
}

\author{
Tursun K. Yuldashev \\ National University of Uzbekistan named after Mirzo Ulugbek, \\ 700174 Tashkent, Uzbekistan \\ tursun.k.yuldashev@gmail.com \\ Bakhtiyor J. Kadirkulov \\ Tashkent State Institute of Oriental Studies, \\ 25 Shaxrisabz, 100047 Tashkent, Uzbekistan \\ kadirkulovbj@gmail.com
}

\begin{abstract}
In this paper, we consider a non-self-adjoint boundary value problem for a fourth-order differential equation of mixed type with Hilfer operator of fractional integro-differentiation in a positive rectangular domain and with spectral parameter in a negative rectangular domain. The mixed type differential equation under consideration is a fourth order differential equation with respect to the second variable. Regarding the first variable, this equation is a fractional differential equation in the positive part of the segment, and is a secondorder differential equation with spectral parameter in the negative part of this segment. A rational method of solving a nonlocal problem with respect to the Hilfer operator is proposed. Using the spectral method of separation of variables, the solution of the problem is constructed in the form of Fourier series. Theorems on the existence and uniqueness of the problem are proved for regular values of the spectral parameter. For sufficiently large positive integers in unique determination of the integration constants in solving countable systems of differential equations, the problem of small denominators arises. Therefore, to justify the unique solvability of this problem, it is necessary to show the existence of values of the spectral parameter such that the quantity we need is separated from zero for sufficiently large $n$. For irregular values of the spectral parameter, an infinite number of solutions in the form of Fourier series are constructed. Illustrative examples are provided.
\end{abstract}

Keywords: Mixed type equation, Non-self-adjoint boundary value problem, Hilfer operator, Mittag-Leffler function, Spectral parameter, Solvability.

\section{Problem statement}

In a rectangular domain $\Omega=\{(t, x):-a<t<b, 0<x<1\}$, we consider the partial differential equation of mixed type

$$
0=\left\{\begin{array}{l}
\left(D^{\alpha, \gamma}+\frac{\partial^{4}}{\partial x^{4}}\right) U(t, x), \quad(t, x) \in \Omega_{1}, \\
\left(\frac{\partial^{2}}{\partial t^{2}}+\omega^{2} \frac{\partial^{4}}{\partial x^{4}}\right) U(t, x), \quad(t, x) \in \Omega_{2}
\end{array}\right.
$$

where $\Omega_{1}=\Omega \cap(t>0), \Omega_{2}=\Omega \cap(t<0)$, $\omega$ is positive spectral parameter, $a$ and $b$ are positive real numbers,

$$
D^{\alpha, \gamma}=J_{0+}^{\gamma-\alpha} \frac{d}{d t} J_{0+}^{1-\gamma} \quad(0<\alpha \leq \gamma \leq 1)
$$


is the Hilfer operator, and

$$
I_{0+}^{\nu} \varphi(t)=\frac{1}{\Gamma(\nu)} \int_{0}^{t}(t-\tau)^{\nu-1} \varphi(\tau) d \tau, \quad \nu>0
$$

is the Riemann-Liouville integral operator [2, pp. 112, 113].

Nonlocal problem. It is required to find a function $U(t, x)$, which belongs to the class

$$
t^{1-\gamma} \frac{\partial^{k} U}{\partial x^{k}} \in C\left(\bar{\Omega}_{1}\right), \quad \frac{\partial^{k} U}{\partial x^{k}} \in C\left(\bar{\Omega}_{2}\right), \quad D^{\alpha, \gamma} U \in C\left(\Omega_{1}\right), \quad U_{t t} \in C\left(\Omega_{2}\right), \quad U_{x x x x} \in C\left(\Omega_{1} \cup \Omega_{2}\right),
$$

$k=\overline{0,3}$ and satisfies the homogeneous equation (1.1) in the domain $\Omega_{1} \cup \Omega_{2}$, the homogeneous boundary value conditions

$$
\left.U\right|_{x=0}=\left.\frac{\partial^{2} U}{\partial x^{2}}\right|_{x=1}=0,\left.\quad \frac{\partial^{k} U}{\partial x^{k}}\right|_{x=0}=\left.\frac{\partial^{k} U}{\partial x^{k}}\right|_{x=1}, \quad k=1,3, \quad t \neq 0,
$$

the nonlocal condition

$$
U(-a, x)=U(b, x)+\varphi(x), \quad 0 \leq x \leq 1,
$$

and the gluing conditions

$$
\lim _{t \rightarrow+0} J_{0+}^{1-\gamma} U(t, x)=\lim _{t \rightarrow-0} U(t, x), \quad \lim _{t \rightarrow+0} J_{0+}^{1-\alpha} \frac{d}{d t} J_{0+}^{1-\gamma} U(t, x)=\lim _{t \rightarrow-0} U_{t}(t, x),
$$

where $\varphi(x)$ is a given sufficiently smooth function.

Let $\left(t_{0} ; b\right) \subset \mathbb{R}^{+} \equiv[0 ; \infty)$ be a finite interval, and let $\alpha>0$. The Riemann-Liouville $\alpha$-order fractional integral of a function $f$ is defined as follows:

$$
I_{t_{0}+}^{\alpha} f(t)=\frac{1}{\Gamma(\alpha)} \int_{t_{0}}^{t}(t-s)^{\alpha-1} f(s) d s, \quad t \in\left(t_{0} ; b\right),
$$

where $\Gamma(\alpha)$ is the Gamma function [2, p. 112].

Let $n-1<\alpha \leq n, n \in \mathbb{N}$. The Riemann-Liouville $\alpha$-order fractional derivative of a function $f$ is defined as follows [9, Vol. 1, p. 27]:

$$
D_{t_{0}+}^{\alpha} f(t)=\frac{d^{n}}{d t^{n}} I_{t_{0}+}^{n-\alpha} f(t), \quad t \in\left(t_{0} ; b\right) .
$$

The Caputo $\alpha$-order fractional derivative of a function $f$ is defined [9, Vol. 1, p. 34] by

$$
{ }_{*} D_{t_{0}+}^{\alpha} f(t)=I_{t_{0}+}^{n-\alpha} f^{(n)}(t)=\frac{1}{\Gamma(n-\alpha)} \int_{t_{0}}^{t} \frac{f^{(n)}(s) d s}{(t-s)^{\alpha-n+1}} \text {. }
$$

Both the derivatives are reduced to the $n$th order derivatives for $\alpha=n \in \mathbb{N}[9$, Vol. 1, pp. 27, 34]:

$$
D_{t_{0}+}^{n} f(t)={ }_{*} D_{t_{0}+}^{n} f(t)=\frac{d^{n} f}{d t^{n}} .
$$

The so-called generalized Riemann-Liouville fractional derivative (referred to as the Hilfer fractional derivative) of order $\alpha, n-1<\alpha \leq n, n \in \mathbb{N}$, and type $\beta, 0 \leq \beta \leq 1$, is defined by the following composition of three operators: [2, p. 113]:

$$
D_{t_{0}+}^{\alpha, \beta} f(t)=I_{t_{0}+}^{\beta(n-\alpha)} \frac{d^{n}}{d t^{n}} I_{t_{0}+}^{(1-\beta)(n-\alpha)} f(t) .
$$


For $\beta=0$, this operator is reduced to the Riemann-Liouville fractional derivative $\left(D_{t_{0}+}^{\alpha, 0}=D_{t_{0}+}^{\alpha}\right)$ and the case $\beta=1$ corresponds to the Caputo fractional derivative: $D_{t_{0}+}^{\alpha, 1}={ }_{*} D_{t_{0}+}^{\alpha}$.

Let $t_{0}=0$ and $\gamma=\alpha+\beta n-\alpha \beta$. It is easy to see that $\alpha \leq \gamma \leq n$. Then it is convenient to use another notation for the operator $D_{0+}^{\alpha, \beta} f(t)$ :

$$
D^{\alpha, \gamma} f(t)=D_{0+}^{\alpha, \beta} f(t) .
$$

For the first time, the generalized Riemann-Liouville operator was introduced in [2] by R. Hilfer on the basis of fractional time evolutions that arise during the transition from the microscopic scale to the macroscopic time scale. Using the integral transforms, he investigated the Cauchy problem for the generalized diffusion equation, the solution of which is presented in the form of the Fox Hfunction. We also note [10,11], where the generalized Riemann-Liouville operator was used in studying dielectric relaxation in glass-forming liquids with different chemical compositions.

In [23], boundary value problems for a fractional diffusion equation with the Hilfer fractional derivative in finite and infinite domains were studied. In the finite domain, the spectral method and the Laplace transform method were used for solving the problem. In the domain infinite with respect to the spatial variable, the Cauchy problem was solved by the Fourier-Laplace integral transform method.

In [12], the properties of the generalized Riemann-Liouville operator were investigated in a special functional space, and an operational method was developed for solving fractional differential equations with this operator. Based on the results of [12], the authors of [15] have developed an operational method for solving fractional differential equations containing a finite linear combination of the generalized Riemann-Liouville operators with various parameters. In [17], the problem of source identification was studied for the generalized diffusion equation with the operator $D^{\alpha, \gamma}$. We also note the work [4], in which inverse problems were investigated for a generalized fourth-order parabolic equation with the operator $D^{\alpha, \gamma}$.

The construction of various models of theoretical physics problems by the aid of fractional calculus is described in [9, Vols. 4, 5], [16, 26]. A specific physical interpretation of the Hilfer fractional derivative, describing the random motion of a particle moving on the real line at Poisson paced times with finite velocity is given in [25]. A detailed review of the application of fractional calculus in solving applied problems is given in [9, Vols. 6-8], [19]. More detailed information as well as a bibliography related to the theory of fractional integro-differentiation, including the Hilfer fractional derivative, can be found in the recently published monograph [24]. In [7], the boundary value problems for the generalized modified moisture transfer equation and difference methods for their numerical implementation were considered.

Nonlocal problems can arise in studying various problems of mathematical biology, predicting soil moisture, problems of plasma. Note that nonlocal conditions of the type (1.3) take place in modeling the problems of the flow around a profile by a subsonic velocity stream with a supersonic zone [20]. More detailed information on nonlocal problems can be found in the monograph [18]. We would like to note some works [14, 30-32], where nonlocal problems for partial differential and integro-differential equations with derivatives of integer or fractional orders were studied.

As for the equations of mixed type, we note the work [8], where I. M. Gel'fand considered an example of gas motion in a channel surrounded by a porous medium, and the gas motion in a channel was described by a wave equation, while the diffusion equation was posed outside the channel. Ya. S. Uflyand considered a problem on the propagation of electric oscillations in compound lines when the losses on a semi-infinite line were neglected and the rest of the line was treated as a cable with no leaks [28]. He reduced this problem to a mixed parabolic-hyperbolic type equation. In [27], a hyperbolic-parabolic system arising in pulse combustion was investigated.

Nonlocal problems for partial differential equations of mixed type were studied by many authors, in particular, in [13, 21, 22, 29, 33]. We would like to note also the results on nonlocal problems 
for parabolic-hyperbolic type equations with fractional order derivatives [1,3]. But these listed works relate mainly to nonlocal problems for fractional mixed type equations of second order. As for mixed fourth-order equations with derivatives of integer or fractional orders, nonlocal problems in such formulation have not been previously studied.

In this paper, we consider a non-self-adjoint boundary value problem for a mixed type fourthorder differential equation with Hilfer operator of fractional integro-differentiation. The spectral method of separation of variables is used taking into account the features of the fractional integrodifferentiation operator. We study the solvability of the nonlocal problem (1.1)-(1.5) for various values of the spectral parameter. This work is a further development and generalization of the results of $[5,6,20]$.

\section{Ordinary differential equation with Hilfer operator}

We consider the Cauchy problem for a differential equation of fractional order with the operator $D^{\alpha, \gamma}$

$$
\left\{\begin{array}{l}
D^{\alpha, \gamma} u(t)=\lambda u(t)+f(t), \quad t \in(0, \ell), \\
\lim _{t \rightarrow+0} J_{0+}^{1-\gamma} u(t)=u_{0}
\end{array}\right.
$$

where $f(t)$ is a given continuous function and $u_{0}=$ const.

Note that the Laplace method was used for solving this problem in [4]. In [15], a solution was found by the operational calculus for a problem more general than (2.1) in a specially constructed functional space. In our work, in contrast to these studies, we use a more rational way to solve problem (2.1), which allows us to obtain an explicit solution.

We prove the following Lemma.

Lemma 1. Assume that $f(t) \in C(0 ; \ell] \cap L_{1}(0 ; \ell)$. Then a solution of problem $u(t) \in C(0 ; \ell] \cap L_{1}(0 ; \ell)$ is representable as follows:

$$
u(t)=u_{0} t^{\gamma-1} E_{\alpha, \gamma}\left(\lambda t^{\alpha}\right)+\int_{0}^{t}(t-\tau)^{\alpha-1} E_{\alpha, \alpha}\left(\lambda(t-\tau)^{\alpha}\right) f(\tau) d \tau
$$

where

$$
E_{\alpha, \beta}(z)=\sum_{k=0}^{\infty} \frac{z^{k}}{\Gamma(\alpha k+\beta)}, \quad z, \alpha, \beta \in \mathbb{C}, \quad \operatorname{Re}(\alpha)>0
$$

is the Mittag-Leffler function [9, Vol. 1, pp. 269-295].

P r o o f. By virtue of the formula (1.6), we rewrite the differential equation of problem (2.1) in the form

$$
J_{0+}^{\gamma-\alpha} D_{0+}^{\gamma} u(t)=\lambda u(t)+f(t) .
$$

Further, applying the operator $J_{0+}^{\alpha}$ to both sides of this equation and taking into account the linearity of this operator and the following formula [15]:

$$
J_{0+}^{\beta} D_{0+}^{\beta} u(t)=u(t)-\left.\frac{1}{\Gamma(\gamma)} J_{0+}^{1-\beta} u(t)\right|_{t=0} t^{\beta-1},
$$

we obtain

$$
u(t)=\frac{u_{0}}{\Gamma(\gamma)} t^{\gamma-1}+J_{0+}^{\alpha} f(t)+\lambda J_{0+}^{\alpha} u(t) .
$$


Using the lemma from [6, p. 123], we represent the solution of equation (2.3) as

$$
\begin{gathered}
u(t)=\frac{u_{0}}{\Gamma(\gamma)} t^{\gamma-1}+J_{0+}^{\alpha} f(t)+ \\
+\lambda \int_{0}^{t}(t-\tau)^{\alpha-1} E_{\alpha, \alpha}\left(\lambda(t-\tau)^{\alpha}\right)\left[\frac{u_{0}}{\Gamma(\gamma)} \tau^{\gamma-1}+J_{0+}^{\alpha} f(\tau)\right] d \tau .
\end{gathered}
$$

We rewrite representation (2.4) as the sum of two expressions $u(t)=I_{1}(t)+I_{2}(t)$, where

$$
\begin{gathered}
I_{1}(t)=u_{0}\left[\frac{t^{\gamma-1}}{\Gamma(\gamma)}+\frac{\lambda}{\Gamma(\gamma)} \int_{0}^{t}(t-\tau)^{\alpha-1} E_{\alpha, \alpha}\left(\lambda(t-\tau)^{\alpha}\right) \tau^{\gamma-1} d \tau\right], \\
I_{2}(t)=J_{0+}^{\alpha} f(t)+\lambda \int_{0}^{t}(t-\tau)^{\alpha-1} E_{\alpha, \alpha}\left(\lambda(t-\tau)^{\alpha}\right) J_{0+}^{\alpha} f(\tau) d \tau .
\end{gathered}
$$

We make the change of variables $s=t-\tau$ in formula (2.5) and use the following formulas [9, Vol. 1 , pp. 269-295]:

$$
\begin{gathered}
E_{\alpha, \mu}(z)=\frac{1}{\Gamma(\mu)}+z E_{\alpha, \mu+\alpha}(t), \quad \alpha>0, \quad \mu>0, \\
\frac{1}{\Gamma(\nu)} \int_{0}^{z}(z-t)^{\nu-1} E_{\alpha, \beta}\left(\lambda t^{\alpha}\right) t^{\beta-1} d t=z^{\beta+\nu-1} E_{\alpha, \beta+\nu}\left(\lambda z^{\alpha}\right), \quad \nu>0, \quad \beta>0 .
\end{gathered}
$$

Then we obtain the following representation for integral (2.5):

$$
I_{1}(t)=u_{0} t^{\gamma-1} E_{\alpha, \gamma}\left(\lambda t^{\alpha}\right) .
$$

The integral in the formula (2.6) is transformed as follows:

$$
\begin{gathered}
\int_{0}^{t}(t-\tau)^{\alpha-1} E_{\alpha, \alpha}\left(\lambda(t-\tau)^{\alpha}\right) J_{0+}^{\alpha} f(\tau) d \tau= \\
=\frac{1}{\Gamma(\alpha)} \int_{0}^{t}(t-\tau)^{\alpha-1} E_{\alpha, \alpha}\left(\lambda(t-\tau)^{\alpha}\right) d \tau \int_{0}^{\tau}(\tau-s)^{\alpha-1} f(s) d s= \\
=\frac{1}{\Gamma(\alpha)} \int_{0}^{t} f(s) d s \int_{s}^{t}(t-\tau)^{\alpha-1}(\tau-s)^{\alpha-1} E_{\alpha, \alpha}\left(\lambda(t-\tau)^{\alpha}\right) d \tau .
\end{gathered}
$$

In view of (2.8), the second integral in the latter equality of formula (2.10) can be written as

$$
\int_{s}^{t}(t-\tau)^{\alpha-1}(\tau-s)^{\alpha-1} E_{\alpha, \alpha}\left(\lambda(t-\tau)^{\alpha}\right) d \tau=\Gamma(\alpha)(t-\tau)^{2 \alpha-1} E_{\alpha, 2 \alpha}\left(\lambda(t-\tau)^{\alpha}\right) .
$$

Then, taking into account (2.7), we represent formula (2.6) in the following form:

$$
I_{2}(t)=\int_{0}^{t}(t-\tau)^{\alpha-1} E_{\alpha, \alpha}\left(\lambda(t-\tau)^{\alpha}\right) f(\tau) d \tau .
$$

Substituting (2.9) and (2.11) into the sum $u(t)=I_{1}(t)+I_{2}(t)$, we obtain formula (2.2). The lemma is proved. 


\section{Uniqueness of solution of the nonlocal problem}

We study this problem by the spectral method of separating variables and seek particular solutions of the nonlocal problem in the form of a product of two functions $U(t, x)=u(t) \cdot \vartheta(x)$. From equation (1.1) and boundary value conditions (1.3), we arrive at the following spectral problem:

$$
\vartheta^{I V}(x)-\lambda^{4} \vartheta(x)=0, \quad \vartheta(0)=\vartheta^{\prime \prime}(1)=0, \quad \vartheta^{\prime}(1)=\vartheta^{\prime}(1), \quad \vartheta^{\prime \prime \prime}(1)=\vartheta^{\prime \prime \prime}(1),
$$

where $\lambda^{4}$ is the constant of separation, $0<\lambda=$ const.

As follows from the results of [5], this spectral problem is non-self-adjoint and has a complete system of eigenfunctions of the following form in the space $L_{2}(0 ; 1)$ :

$$
\begin{gathered}
\vartheta_{0}(x)=2 x, \quad \vartheta_{n 1}(x)=2 \sin \lambda_{n} x, \quad \vartheta_{n 2}(x)=\frac{e^{\lambda_{n} x}-e^{\lambda_{n}(1-x)}}{e^{\lambda_{n}}-1}+\cos \lambda_{n} x, \\
\lambda_{n}=2 \pi n, \quad n \in \mathbb{N} .
\end{gathered}
$$

System (3.1) forms a Riesz basis in $L_{2}(0 ; 1)$. In [5], it was also proved that there exists a biorthogonal system of functions with (3.1):

$$
\eta_{0}(x)=1, \quad \eta_{n 1}(x)=\frac{e^{\lambda_{n} x}+e^{\lambda_{n}(1-x)}}{e^{\lambda_{n}}-1}+\sin 2 \pi n x, \quad \eta_{n 2}(x)=2 \cos \lambda_{n} x .
$$

System (3.2) also forms a Riesz basis in $L_{2}(0 ; 1)$.

Let $U(t, x)$ be a solution of the nonlocal problem. We consider the functions

$$
\begin{gathered}
u_{0}^{+}(t)=\int_{0}^{1} U(t, x) d x, \quad u_{n i}^{+}(t)=\int_{0}^{1} U(t, x) \eta_{n i}(x) d x, \quad t>0, \\
u_{0}^{-}(t)=\int_{0}^{1} U(t, x) d x, \quad u_{n i}^{-}(t)=\int_{0}^{1} U(t, x) \eta_{n i}(x) d x, \quad i=1,2, \quad t<0,
\end{gathered}
$$

where the functions $\eta_{0}(x)$ and $\eta_{n i}(x), i=1,2$, are defined in (3.2).

Applying the operator $D^{\alpha, \gamma}$ with respect to $t$ to both sides of equality (3.3), differentiating (3.4) twice with respect to $t$, and taking into account equation (1.1), we obtain differential equations with respect to the functions $u_{0}^{ \pm}(t)$ and $u_{n i}^{ \pm}(t), i=1,2$ :

$$
\begin{aligned}
& D^{\alpha, \gamma} u_{0}^{+}(t)=0, \quad D^{\alpha, \gamma} u_{n i}^{+}(t)+\lambda_{n}^{4} u_{n i}^{+}(t)=0, \quad i=1,2, \quad t>0, \\
& \frac{d^{2}}{d t^{2}} u_{0}^{-}(t)=0, \quad \frac{d^{2}}{d t^{2}} u_{n i}^{-}(t)+\lambda_{n}^{4} \omega^{2} u_{n i}^{-}(t)=0, \quad i=1,2, \quad t<0 .
\end{aligned}
$$

The general solutions of these differential equations (3.5) and (3.6) have the form

$$
u_{0}^{ \pm}(t)=\left\{\begin{array}{ll}
\frac{A_{0}}{\Gamma(\gamma)} t^{\gamma-1}, & t>0, \\
B_{0} t+C_{0}, & t<0,
\end{array} \quad u_{n i}^{ \pm}(t)=\left\{\begin{array}{l}
A_{n i} t^{\gamma-1} E_{\alpha, \gamma}\left(-\lambda_{n}^{4} t^{\alpha}\right), \quad t>0, \\
B_{n i} \sin \lambda_{n}^{2} \omega t+C_{n i} \cos \lambda_{n}^{2} \omega t, \quad t<0,
\end{array}\right.\right.
$$

where $A_{0}, B_{0}, C_{0}, A_{n i}, B_{n i}$, and $C_{n i}$ are arbitrary constants, $i=1,2, n=1,2, \ldots$.

Taking into account conditions (1.4) and (1.5), we conclude from (3.3) and (3.4) that the functions $u_{0}^{ \pm}(t)$ and $u_{n i}^{ \pm}(t), \quad i=1,2$, in (3.7) must satisfy the following conditions:

$$
\lim _{t \rightarrow+0} J_{0+}^{1-\gamma} u_{0}^{+}(t)=\lim _{t \rightarrow-0} u_{0}^{-}(t), \quad \lim _{t \rightarrow+0} J_{0+}^{1-\alpha}\left(\frac{d}{d t} J_{0+}^{1-\gamma} u_{0}^{+}(t)\right)=\lim _{t \rightarrow-0} \frac{d u_{0}^{-}(t)}{d t}
$$




$$
\begin{gathered}
\lim _{t \rightarrow+0} J_{0+}^{1-\gamma} u_{n i}^{+}(t)=\lim _{t \rightarrow-0} u_{n i}^{-}(t), \quad \lim _{t \rightarrow+0} J_{0+}^{1-\alpha}\left(\frac{d}{d t} J_{0+}^{1-\gamma} u_{n i}^{+}(t)\right)=\lim _{t \rightarrow-0} \frac{d u_{n i}^{-}(t)}{d t}, \\
u_{0}^{-}(-a)=u_{0}^{+}(b)+\varphi_{0}, \quad u_{n i}^{-}(-a)=u_{n i}^{+}(b)+\varphi_{n i}, \quad i=1,2
\end{gathered}
$$

where

$$
\varphi_{0}=\int_{0}^{1} \varphi(x) d x, \quad \varphi_{n i}=\int_{0}^{1} \varphi(x) \eta_{n i}(x) d x, \quad i=1,2, \quad n=1,2, \ldots
$$

Therefore, we obtain the following systems of algebraic equations:

$$
\begin{gathered}
\left\{\begin{array}{l}
A_{0}=C_{0}, \quad B_{0}=0, \\
-B_{0} a+C_{0}=\frac{A_{0}}{\Gamma(\gamma)} b^{\gamma-1}+\varphi_{0},
\end{array}\right. \\
\left\{\begin{array}{l}
A_{n i}=C_{n i}, \quad \omega B_{n i}=-\lambda_{n}^{2} A_{n i}, \\
-B_{n i} \sin \lambda_{n}^{2} \omega a+C_{n i} \cos \lambda_{n}^{2} \omega a-A_{n i} b^{\gamma-1} E_{\alpha, \gamma}\left(-\lambda_{n}^{4} b^{\alpha}\right)=\varphi_{n i} .
\end{array}\right.
\end{gathered}
$$

Each of systems (3.11) and (3.12) has a unique solution

$$
C_{0}=A_{0}, \quad B_{0}=0, \quad A_{0}=\frac{\varphi_{0}}{\Delta_{0}}, \quad C_{n i}=A_{n i}=\frac{\varphi_{n i}}{\Delta_{n}(\omega)}, \quad B_{n i}=-\frac{\lambda_{n}^{2}}{\omega} \frac{\varphi_{n i}}{\Delta_{n}(\omega)},
$$

if the following condition holds for all $n \in \mathbb{N}_{0}=\mathbb{N} \cup\{0\}$ :

$$
\Delta_{n}(\omega)=\lambda_{n}^{2} \omega \sin \lambda_{n}^{2} \omega a+\cos \lambda_{n}^{2} \omega a-b^{\gamma-1} E_{\alpha, \gamma}\left(-\lambda_{n}^{4} b^{\alpha}\right) \neq 0 .
$$

Substituting (3.13) into (3.7), we obtain the representation

$$
\begin{gathered}
u_{0}^{ \pm}(t)=\left\{\begin{array}{l}
\frac{\varphi_{0}}{\Gamma_{0}(\gamma) \Delta_{0}} t^{\gamma-1}, \quad t>0, \\
\frac{\varphi_{0}}{\Delta_{0}}, \quad t \leq 0,
\end{array}\right. \\
u_{n i}^{ \pm}(t)=\left\{\begin{array}{l}
\frac{\varphi_{n i}}{\Delta_{n}(\omega)} t^{\gamma-1} E_{\alpha, \gamma}\left(-\lambda_{n}^{4} t^{\alpha}\right), \quad t>0, \\
\frac{\varphi_{n i}}{\Delta_{n}(\omega)}\left(\cos \lambda_{n}^{2} \omega t-\frac{\lambda_{n}^{2}}{\omega} \sin \lambda_{n}^{2} \omega t\right), \quad t \leq 0 .
\end{array}\right.
\end{gathered}
$$

We show the uniqueness of the solution of the nonlocal problem under condition (3.14). Suppose the opposite. Let the nonlocal problem have two different solutions $U_{1}(t, x)$ and $U_{2}(t, x)$, and let $U(t, x)=U_{1}(t, x)-U_{2}(t, x)$. It is not difficult to see that $U(t, x)$ is a solution of the homogeneous nonlocal problem $(\varphi(x)=0)$. This is why one only needs to prove that the homogeneous problem has only the trivial solution.

Suppose that condition (3.14) holds and $\varphi(x) \equiv 0$. Then $\varphi_{0}=0, \varphi_{n i}=0, i=1,2$, and the representations (3.3), (3.4) and (3.15), (3.16) yield

$$
\begin{aligned}
& \int_{0}^{1} t^{1-\gamma} U(t, x) d x=0, \quad \int_{0}^{1} t^{1-\gamma} U(t, x) \eta_{n i}(x) d x=0, \quad t \in[0 ; b], \\
& \int_{0}^{1} U(t, x) d x=0, \quad \int_{0}^{1} U(t, x) \eta_{n i}(x) d x=0, \quad t \in[-a ; 0], \quad i=1,2 .
\end{aligned}
$$


Further, taking into account the completeness of system (3.2) in the space $L_{2}(0 ; 1)$, we conclude that $U(t, x)=0$ almost everywhere on $[0 ; 1]$ for all $t \in[-a ; b]$. Since $t^{1-\gamma} U(t, x) \in C\left(\bar{\Omega}_{1}\right)$ and $U(t, x) \in C\left(\bar{\Omega}_{2}\right)$, we have $t^{1-\gamma} U(t, x) \equiv 0$ in the domain $\bar{\Omega}$. Therefore, the solution of the nonlocal problem is unique in the domain $\bar{\Omega}$.

Thus, we have proved the following theorem.

Theorem 1. Suppose that there exists a solution of the nonlocal problem. This solution is unique if condition (3.14) holds for all $n \in \mathbb{N}_{0}$.

\section{Existence of a solution of the nonlocal problem}

Now we consider the case when condition (3.14) is violated. Let $\Delta_{m}(\omega)=0$ for all $\omega, \gamma \in(0 ; 1)$ and $n=m$. Then the homogeneous nonlocal problem $(\varphi(x) \equiv 0)$ has a nontrivial solution

$$
V_{m i}^{ \pm}(t, x)=v_{m}^{ \pm}(t) \vartheta_{m i}(x), \quad i=1,2,
$$

where

$$
v_{m}^{ \pm}(t)=\left\{\begin{array}{l}
t^{\gamma-1} E_{\alpha, \gamma}\left(-\lambda_{m}^{4} t^{\alpha}\right), \quad t>0, \\
\cos \lambda_{m}^{2} \omega t-\frac{\lambda_{n}^{2}}{\omega} \sin \lambda_{m}^{2} \omega t, \quad t<0 .
\end{array}\right.
$$

It is easy to verify that, for $\gamma=1$, the function $V(t, x)=x$ is also a nontrivial solution of the homogeneous nonlocal problem.

From $\Delta_{n}(\omega)=0$, we come to the trigonometric equation

$$
\sqrt{1+\omega^{2} \lambda_{n}^{4}} \sin \left(\lambda_{n}^{2} \omega a+\rho_{n}\right)-b^{\gamma-1} E_{\alpha, \gamma}\left(-\lambda_{n}^{4} b^{\alpha}\right)=0,
$$

where $\rho_{n}=\arcsin \left(1 / \sqrt{1+\omega^{2} \lambda_{n}^{4}}\right)$ and $\rho_{n} \rightarrow 0$ as $n \rightarrow+\infty$. Hence, we conclude that the expression $\Delta_{n}(\omega)$ is zero only if

$$
\omega=\frac{1}{\lambda_{n}^{2} a}\left[(-1)^{k} \arcsin \frac{b^{\gamma-1} E_{\alpha, \gamma}\left(-\lambda_{n}^{4} b^{\alpha}\right)}{\sqrt{1+\omega^{2} \lambda_{n}^{4}}}+\pi k-\rho_{n}\right], \quad k=1,2, \ldots
$$

The set $\Im$ of positive solutions of trigonometric equation (4.2) is called the set of irregular values of the spectral parameter $\omega$.

The set of remaining values of the spectral parameter $\aleph=(0 ; \infty) \backslash \Im$ is called the set of regular values of the spectral parameter $\omega$.

Since $\Delta_{n}(\omega)$ is the denominator of a fraction and its values can become quite small for sufficiently large $n$, the problem of "small denominators" arises. Therefore, in order to justify the unique solvability of the nonlocal problem for regular values of the spectral parameter $\omega$, it is necessary to show that the quantity $\Delta_{n}(\omega)$ is separated from zero for sufficiently large $n$.

Lemma 2. Suppose that $\gamma \in(0 ; 1], a$ and $b$ are arbitrary positive real numbers, and $\omega$ is such that the product $\pi \omega$ a is a rational number. Then, for large $n$, there exists a positive constant $M_{0}$ such that the following estimate holds:

$$
\left|\Delta_{n}(\omega)\right| \geq M_{0}>0
$$

P r o o f. I. We set $\omega=p / \pi a, p \in \mathbb{N}$. Then we derive from (4.2) that, for all $n$ and $a, b>0$,

$$
\left|\Delta_{n}(\omega)\right| \geq\left| \pm \sqrt{1+16 n^{4} \pi^{2} \frac{p^{2}}{a^{2}}}-b^{\gamma-1} E_{\alpha, \gamma}\left(-16 n^{4} \pi^{4} b^{\alpha}\right)\right| \geq
$$




$$
\geq\left|1-b^{\gamma-1} E_{\alpha, \gamma}\left(-16 n^{4} \pi^{4} b^{\alpha}\right)\right| \geq 1-b^{\gamma-1} E_{\alpha, \gamma}\left(-16 n^{4} \pi^{4} b^{\alpha}\right) .
$$

We use the following properties of the Mittag-Leffler function [9, Vol. 1, pp. 269-295].

(1) For all $\lambda>0, \alpha, \gamma \in(0 ; 1], \alpha \leq \gamma$, and $t>0$, the function $t^{\alpha-1} E_{\alpha, \gamma}\left(-\lambda t^{\alpha}\right)$ is completely monotone, i.e.,

$$
(-1)^{n}\left[t^{\gamma-1} E_{\alpha, \gamma}\left(-\lambda t^{\alpha}\right)\right]^{(n)} \geq 0, \quad n=0,1,2, \ldots .
$$

(2) The following estimate is true for all $\alpha \in(0 ; 2), \gamma \in \mathbb{R}$, and $\arg z=\pi$ :

$$
\left|E_{\alpha, \gamma}(z)\right| \leq \frac{M}{1+|z|}
$$

where $0<M=$ const is independent of $z$.

Then, (4.4) implies that there exists a number $n_{0} \in \mathbb{N}$ such that for all $n>n_{0}$ we have

$$
1-b^{\gamma-1} E_{\alpha, \gamma}\left(-16 n^{4} \pi^{4} b^{\alpha}\right)=M_{1}>0 .
$$

Consequently, $\Delta_{n}(\omega) \geq M_{1}>0$.

II. Now we set

$$
\frac{p}{q}=4 \pi \omega a \in \mathbb{Q} \Leftrightarrow \omega=\frac{1}{4 \pi a} \frac{p}{q},
$$

where $p, q \in \mathbb{N},(p, q)=1$. We divide $n^{2} p$ by $q$ with a remainder: $n^{2} p=s q+r, s \in \mathbb{N}, 0 \leq r<q$. Then from (4.1), we obtain

$$
\left|\Delta_{n}(\omega)\right|=\left|\sqrt{1+\left[\frac{\pi}{a}\left(s+\frac{r}{q}\right)\right]^{2}}(-1)^{s} \sin \left(\frac{\pi r}{q}+\rho_{n}\right)-b^{\gamma-1} E_{\alpha, \gamma}\left(-16 n^{4} \pi^{4} b^{\alpha}\right)\right| .
$$

If $r=0$, then this case reduces to case I.

Suppose that $r>0$. Since $\rho_{n} \rightarrow 0$ as $n \rightarrow+\infty$, there exists a number $n_{1}>0$ such that $\rho_{n}<\pi /(2 q)$ for all $n>n_{1}$. Thus, we obtain the lower estimate

$$
\begin{gathered}
\left|\Delta_{n}(\omega)\right| \geq\left|\sqrt{1+\left[\frac{\pi}{a}\left(s+\frac{r}{q}\right)\right]^{2}} \sin \left(\frac{\pi r}{q}+\rho_{n}\right)-b^{\gamma-1} E_{\alpha, \gamma}\left(-16 n^{4} \pi^{4} b^{\alpha}\right)\right| \geq \\
\geq \sqrt{1+\left[\frac{\pi}{a}\left(s+\frac{r}{q}\right)\right]^{2}}\left|\sin \left(\frac{\pi r}{q}+\rho_{n}\right)\right|-b^{\gamma-1} E_{\alpha, \gamma}\left(-16 n^{4} \pi^{4} b^{\alpha}\right)> \\
>\frac{\pi}{a}\left(s+\frac{r}{q}\right)\left|\sin \left(\frac{\pi(q-1)}{q}+\frac{\pi}{2 q}\right)\right|-1=\frac{\pi}{a}\left(s+\frac{r}{q}\right) \sin \frac{\pi}{2 q}-1=M_{2}>0
\end{gathered}
$$

for

$$
n_{2} \geq\left[a q\left(\pi p \sin \frac{\pi}{2 q}\right)^{-1}\right]^{1 / 2}
$$

Setting $M_{0}>\max \left\{M_{1}, M_{2}\right\}$ and $n>\max \left\{n_{0}, n_{1}, n_{2}\right\}$, we complete the proof of the lemma. Lemma 2 is proved.

We call the solution of the nonlocal problem (1.1)-(1.5) for regular values of the spectral parameter $\omega$ a regular solution of the nonlocal problem. Estimates (4.3) and (4.5) imply the following lemma. 
Lemma 3. The following estimates hold for regular values of the spectral parameter $\omega$ :

$$
\begin{gathered}
t^{1-\gamma}\left|u_{0}^{+}(t)\right| \leq C_{1}\left|\varphi_{0}\right|, \quad t^{1-\gamma}\left|u_{n i}^{+}(t)\right| \leq C_{2}\left|\varphi_{n i}\right|, \\
t^{1-\gamma}\left|D^{\alpha, \beta} u_{n i}^{+}(t)\right| \leq C_{3} n^{4}\left|\varphi_{n i}\right|, \quad i=1,2, \quad t \in[0 ; b] ; \\
\left|u_{0}^{-}(t)\right| \leq C_{4}\left|\varphi_{0}\right|, \quad\left|u_{n i}^{-}(t)\right| \leq C_{5} n^{2}\left|\varphi_{n i}\right|, \\
\left|\frac{d u_{n i}^{-}(t)}{d t}\right| \leq C_{6} n^{4}\left|\varphi_{n i}\right|, \quad\left|\frac{d^{2} u_{n i}^{-}(t)}{d t^{2}}\right| \leq C_{7} n^{6}\left|\varphi_{n i}\right|, \quad i=1,2, \quad t \in[-a ; 0],
\end{gathered}
$$

where $C_{k}, k=\overline{1,7}$, are positive constants.

Since system (3.1) is complete and forms a Riesz basis in $L_{2}(0 ; 1)$, we write the solution of the nonlocal problem for regular values of the spectral parameter $\omega$ as

$$
U(t, x)= \begin{cases}u_{0}^{+}(t) \vartheta_{0}(x)+\sum_{n=1}^{\infty} \sum_{i=1}^{2} u_{n i}^{+}(t) \vartheta_{n i}(x), & (t, x) \in \Omega_{1}, \\ u_{0}^{-}(t) \vartheta_{0}(x)+\sum_{n=1}^{\infty} \sum_{i=1}^{2} u_{n i}^{-}(t) \vartheta_{n i}(x), & (t, x) \in \Omega_{2},\end{cases}
$$

where $u_{0}^{ \pm}(t), u_{n 1}^{ \pm}(t)$, and $u_{n 2}^{ \pm}(t)$ are defined in (3.15) and (3.16).

Indeed, substituting function (4.6) into the mixed equation (1.1) and satisfying conditions (1.3)-(1.5), we obtain problems (3.5), (3.6), (3.8)-(3.10) with respect to the desired functions. The solutions of these problems can be represented as functions (3.15) and (3.16).

Now formally differentiating term-by-term the series (4.6) the required number of times, we obtain the series

$$
\begin{gathered}
D^{\alpha, \gamma} U(t, x)=\sum_{n=1}^{\infty} \sum_{i=1}^{2} D^{\alpha, \gamma} u_{n i}^{+}(t) \vartheta_{n i}(x), \quad t>0, \\
\frac{\partial^{k} U(t, x)}{\partial x^{k}}=u_{0}^{+}(t) \frac{d^{k} \vartheta_{0}(x)}{d x^{k}}+\sum_{n=1}^{\infty} \sum_{i=1}^{2} u_{n i}^{+}(t) \frac{d^{k} \vartheta_{n i}(x)}{d x^{k}}, \quad k=\overline{1,4}, \quad t>0, \\
\frac{\partial^{2} U(t, x)}{\partial t^{2}}=\sum_{n=1}^{\infty} \sum_{i=0}^{2} \frac{d^{2} u_{n i}^{-}(t)}{d t^{2}} \vartheta_{n i}(x), \quad t<0, \\
\frac{\partial^{k} U(t, x)}{\partial x^{k}}=u_{0}^{-}(t) \frac{d^{k} \vartheta_{0}(x)}{d x^{k}}+\sum_{n=1}^{\infty} \sum_{i=1}^{2} u_{n 2}^{-}(t) \frac{d^{k} \vartheta_{n 2}(x)}{d x^{k}}, \quad k=\overline{0,4}, \quad t<0 .
\end{gathered}
$$

By virtue of Lemma 2 and Lemma 3, we conclude that series (4.9) and (4.10) are majorized by the following sum of series:

$$
\sum_{n=1}^{\infty} n^{6}\left|\varphi_{n 1}\right|+\sum_{n=1}^{\infty} n^{6}\left|\varphi_{n 2}\right|
$$

Multiplying series (4.7) and (4.8) term-by-term by $t^{1-\gamma}$, we obtain the series

$$
\sum_{n=1}^{\infty} \sum_{i=1}^{2} t^{1-\gamma} D^{\alpha, \gamma} u_{n i}^{+}(t) \vartheta_{n i}(x), \quad \sum_{n=1}^{\infty} \sum_{i=1}^{2} t^{1-\gamma} u_{n i}^{+}(t) \frac{d^{k} \vartheta_{n i}(x)}{d x^{k}}, \quad k=\overline{0,4}, \quad t>0 .
$$


The series in (4.12) are also majorized by the series (4.11). Taking into account the fact that the function $\varphi(x)$ is sufficiently smooth and integrating by parts

$$
\varphi_{n i}=\int_{0}^{1} \varphi(x) \eta_{n i}(x) d x, \quad i=1,2
$$

we derive

$$
\begin{gathered}
\varphi_{n 1}=-\frac{1}{(2 \pi n)^{7}} \varphi_{n 1}^{(7)}=-\frac{1}{(2 \pi n)^{7}}\left(\varphi^{(7)}(x), \vartheta_{n 2}(x)\right), \\
\varphi_{n 2}=\frac{1}{(2 \pi n)^{7}} \varphi_{n 2}^{(7)}=\frac{1}{(2 \pi n)^{7}}\left(\varphi^{(7)}(x), \vartheta_{n 1}(x)\right) .
\end{gathered}
$$

By virtue of these representations, we apply the Cauchy-Schwartz inequality and Bessel inequality to (4.11)

$$
\sum_{n=1}^{\infty} n^{6}\left|\varphi_{n i}\right| \leq \sum_{n=1}^{\infty} \frac{1}{n}\left|\varphi_{n i}^{(7)}\right| \leq\left(\sum_{n=1}^{\infty} \frac{1}{n^{2}}\right)^{1 / 2}\left(\sum_{n=1}^{\infty}\left|\varphi_{n i}^{(7)}\right|^{2}\right)^{1 / 2} \leq C\left\|\varphi^{(7)}(x)\right\|_{L_{2}(0,1)}<\infty, \quad i=1,2 .
$$

This estimate implies that series (4.9) and (4.10) converge absolutely and uniformly in the domains $\bar{\Omega}_{1}$ and $\bar{\Omega}_{2}$, respectively. Therefore, the function $U(t, x)$, represented by series (4.6), possesses properties (1.2) and satisfies conditions (1.3)-(1.5).

We note that $\Delta_{n}(\omega)=0$ for irregular values of the spectral parameter $\omega$ and $n=k_{1}, \ldots, k_{s}$, $1 \leq k_{1}<k_{1}<\cdots<k_{s}, s \in \mathbb{N}(\gamma \neq 1)$. Then, the following orthogonality conditions are necessary and sufficient for the solvability of systems (3.11) and (3.12):

$$
\varphi_{n i}=\int_{0}^{1} \varphi(x) \eta_{n i} d x=0, \quad i=1,2, \quad n=k_{1}, \ldots, k_{s} .
$$

In this case, the solutions of the nonlocal problem are representable as a sum of series

$$
\begin{gathered}
U(t, x)=u_{0}^{ \pm}(t) \vartheta_{0}(x)+ \\
+\left[\sum_{n=1}^{k_{1}-1}+\sum_{n=k_{1}+1}^{k_{2}-1}+\cdots+\sum_{n=k_{s}+1}^{\infty}\right] \sum_{i=1}^{2} u_{n i}^{ \pm}(t) \vartheta_{n i}(x)+\sum_{m} \sum_{i=1}^{2} C_{m i} V_{m i}^{ \pm}(t),
\end{gathered}
$$

where $m=k_{1}, \ldots, k_{s}, C_{m i}$ are arbitrary constants, and the functions $V_{m i}^{ \pm}(t), i=1,2$, are defined in (4.1). Note that, in the case $\gamma=1$, we replace the function $u_{0}^{ \pm}(t)$ in (4.14) with a constant $C_{0}$; moreover, the orthogonality condition

$$
\varphi_{0}=\int_{0}^{1} \varphi(x) d x=0
$$

is added to formula (4.13).

Thus, the following theorem is proved.

Theorem 2. Suppose that the following conditions are fulfilled:

$$
\begin{gathered}
\varphi(x) \in C^{6}[0 ; 1], \quad \varphi^{(7)}(x) \in L_{2}(0 ; 1), \quad \varphi^{(2 k)}(0)=0, \\
\varphi^{(2(k+1))}(1)=0, \quad k=\overline{0,2}, \quad \varphi^{(k)}(0)=\varphi^{(k)}(1), \quad k=1,3,5 .
\end{gathered}
$$


Then the nonlocal boundary value problem is uniquely solvable for regular values of the spectral parameter $\omega$, and this solution is represented in the form of the Fourier series (4.6) in the domain $\Omega$.

For irregular values of the spectral parameter $\omega$ and some $n=k_{1}, \ldots, k_{s}$, the nonlocal problem has an infinite number of solutions in the form of series (4.14).

For $\gamma<1$, the solvability condition has the form (4.13). For $\gamma=1$ in (4.14), the function $u_{0}^{ \pm}(t)$ is replaced with a constant $C_{0}$ and conditions (4.13), and (4.15) are the solvability conditions.

\section{Stability of solution of the nonlocal problem}

For regular values of the spectral parameter $\omega$, we consider the question of the stability of the solution of the nonlocal problem with respect to the function $\varphi(x)$ from condition (1.4). To this end, we introduce the norm in the space of continuous functions as follows:

$$
\begin{gathered}
\|U(t, x)\|_{C(\bar{\Omega})}=\left\|t^{1-\gamma} U(t, x)\right\|_{C\left(\bar{\Omega}_{1}\right)}+\|U(t, x)\|_{C\left(\bar{\Omega}_{2}\right)}= \\
=\max _{(t, x) \in \bar{\Omega}_{1}}\left|t^{1-\gamma} U(t, x)\right|+\max _{(t, x) \in \bar{\Omega}_{1}}|U(t, x)| .
\end{gathered}
$$

Theorem 3. Suppose that all the conditions of Theorem 2 are fulfilled. Then the following estimate holds for the solution of the nonlocal problem with regular values of the spectral parameter $\omega$ :

$$
\|U(t, x)\|_{C(\bar{\Omega})} \leq C\left\|\varphi^{\prime \prime \prime}(x)\right\|_{C[0 ; 1]}
$$

where $0<C=$ const is independent of $\varphi(x)$ and $\|f(x)\|_{C[0 ; 1]}=\max _{[0 ; 1]}|f(x)|$.

P r o o f. Let $(t, x)$ be an arbitrary point of the domain $\bar{\Omega}_{2}$. Then we have the representations

$$
\begin{gathered}
\varphi_{n 1}=-\frac{1}{\lambda_{n}^{3}} \varphi_{n 1}^{(3)}, \quad \varphi_{n 1}^{(3)}=\int_{0}^{1} \varphi^{\prime \prime \prime}(x) \vartheta_{n 2}(x) d x, \\
\varphi_{n 2}=\frac{1}{\lambda_{n}^{3}} \varphi_{n 2}^{(3)}, \quad \varphi_{n 2}^{(3)}=\int_{0}^{1} \varphi^{\prime \prime \prime}(x) \vartheta_{n 1}(x) d x .
\end{gathered}
$$

Applying Lemma 3 and the Cauchy-Schwarz inequality to (4.6), we obtain

$$
\begin{aligned}
& \|U(t, x)\|_{C\left(\bar{\Omega}_{2}\right)} \leq 2 C_{4}\left|\varphi_{0}\right|+C_{5} \sum_{n=1}^{\infty} \frac{1}{n}\left(\left|\varphi_{n 1}^{(3)}\right|+\left|\varphi_{n 2}^{(3)}\right|\right) \leq \\
& \leq 2 C_{4}\left|\varphi_{0}\right|+C_{5}\left(\sum_{n=1}^{\infty} \frac{1}{n^{2}}\right)^{1 / 2}\left(\sum_{n=1}^{\infty}\left(\left|\varphi_{n 1}^{(3)}\right|+\left|\varphi_{n 2}^{(3)}\right|\right)^{2}\right)^{1 / 2} .
\end{aligned}
$$

It is well known that the former series converges. Applying the inequality $(|a|+|b|)^{2} \leq 2\left(|a|^{2}+|b|^{2}\right)$ and the Bessel inequality to the latter series, we obtain

$$
\sum_{n=1}^{\infty}\left(\left|\varphi_{n 1}^{(3)}\right|+\left|\varphi_{n 2}^{(3)}\right|\right)^{2} \leq 2 \sum_{n=1}^{\infty}\left(\left|\varphi_{n 1}^{(3)}\right|^{2}+\left|\varphi_{n 2}^{(3)}\right|^{2}\right) \leq C_{11}\left\|\varphi^{\prime \prime \prime}(x)\right\|_{L_{2}(0 ; 1)}^{2}, \quad 0<C_{11}=\text { const. }
$$

Similarly, we can find for all $(t, x) \in \bar{\Omega}_{1}$ that

$$
\left\|t^{1-\gamma} U(t, x)\right\|_{C\left(\bar{\Omega}_{1}\right)} \leq C_{12}\left\|\varphi^{\prime \prime \prime}(x)\right\|_{L_{2}(0 ; 1)}^{2}, \quad 0<C_{12}=\text { const. }
$$

Estimates (6.1) and (6.2) imply estimate (5.1), where $C=C_{11}+C_{12}$. If we assume that $\left\|\varphi^{\prime \prime \prime}(x)\right\|_{L_{2}(0 ; 1)}^{2}<\delta$, then the estimate $\|U(t, x)\|_{C(\bar{\Omega})}<\varepsilon$ is true for all $\varepsilon=C \cdot \delta$. The theorem is proved. 


\section{Illustrative examples}

Example 1. Consider the nonlocal problem for $\gamma=1$. Then we have $D^{\alpha, \gamma}=D^{\alpha, 1}={ }_{C} D^{\alpha}$ and equation (1.1) takes the form

$$
0= \begin{cases}{ }_{C} D^{\alpha} U(t, x)+\frac{\partial^{4} U(t, x)}{\partial x^{4}}, & t>0, \\ \frac{\partial^{2} U(t, x)}{\partial t^{2}}+\omega^{2} \frac{\partial^{4} U(t, x)}{\partial x^{4}}, & t<0 .\end{cases}
$$

Equations (6.1) is a mixed type differential equation with the Caputo operator in a positive rectangular domain. We consider it under conditions (1.3)-(1.5). From (3.14), we obtain $A_{0}=\varphi_{0}=0$, i.e., we arrive at condition (4.15). The solution of this problem with regular values of the spectral parameter $\omega$ can be represented as

$$
U(t, x)=\left\{\begin{array}{l}
\sum_{n=1}^{\infty} \sum_{i=1}^{2} \frac{\varphi_{n i}}{\Delta_{n}(\omega)} E_{\alpha, 1}\left(-\lambda_{n}^{4} t^{\alpha}\right) \vartheta_{n i}(x)+C_{01} x, \quad(t, x) \in \Omega_{1}, \\
\sum_{n=1}^{\infty} \sum_{i=1}^{2} \frac{\varphi_{n i}}{\Delta_{n}(\omega)}\left(\cos \lambda_{n}^{2} \omega t-\frac{\lambda_{n}^{2}}{\omega} \sin \lambda_{n}^{2} \omega t\right) \vartheta_{n i}(x)+C_{02} x, \quad(t, x) \in \Omega_{2},
\end{array}\right.
$$

where $C_{0 i}=$ const, $i=1,2$.

Example 2. Consider the nonlocal problem for $\gamma=\alpha<1$. Then we have $D^{\alpha, \gamma}=D^{\alpha, \alpha}={ }_{R L} D^{\alpha}$ and equation (1.1) takes the form

$$
0= \begin{cases}R L D^{\alpha} U(t, x)+\frac{\partial^{4} U(t, x)}{\partial x^{4}}, & t>0, \\ \frac{\partial^{2} U(t, x)}{\partial t^{2}}+\omega^{2} \frac{\partial^{4} U(t, x)}{\partial x^{4}}, & t<0 .\end{cases}
$$

Equation (6.2) is a mixed type differential equation with the Riemann-Liouville operator in a positive rectangular domain. We consider it under conditions (1.3)-(1.5). A solution of this problem with regular values of the spectral parameter $\omega$ exists and is unique. This solution has a representation coinciding with (4.6) for $\gamma=\alpha<1$.

Example 3. Consider the case $\gamma=\alpha=1$. Then we have $D^{\alpha, \gamma}=D^{1,1}=d / d t$ and equation (1.1) takes the form

$$
0=\left\{\begin{array}{l}
\frac{\partial U(t, x)}{\partial t}+\frac{\partial^{4} U(t, x)}{\partial x^{4}}, \quad t>0, \\
\frac{\partial^{2} U^{4}(t, x)}{\partial t^{2}}+\omega^{2} \frac{\partial^{4} U(t, x)}{\partial x^{4}}, \quad t<0 .
\end{array}\right.
$$

We obtained a mixed type differential equation of integer order, which is a particular case of equation (6.1) and, therefore, the solvability condition for this problem coincides with condition (4.15), and the solution of the nonlocal problem is represented as

$$
U(t, x)=\left\{\begin{array}{l}
\sum_{n=1}^{\infty} \sum_{i=1}^{2} \frac{\varphi_{n i}}{\Delta_{n}(\omega)} e^{-\lambda_{n}^{4} t} \vartheta_{n i}(x)+A x, \quad(t, x) \in \Omega_{1}, \\
\sum_{n=1}^{\infty} \sum_{i=1}^{2} \frac{\varphi_{n i}}{\Delta_{n}(\omega)}\left(\cos \lambda_{n}^{2} \omega t-\frac{\lambda_{n}^{2}}{\omega} \sin \lambda_{n}^{2} \omega t\right) \vartheta_{n i}(x)+A x, \quad(t, x) \in \Omega_{2},
\end{array}\right.
$$

where $A=$ const. 


\section{Conclusion}

We established a criterion for the existence and uniqueness of the regular solution of the nonlocal problem for a fourth-order differential equation of mixed type with Hilfer operator in a positive rectangular domain and with spectral parameter in a negative rectangular domain. We use the spectral method of separation of variables, which helps us to construct the solution of the nonlocal problem (1.1)-(1.5) in the form of Fourier series. Theorems on the existence and uniqueness of the problem are proved for regular values of the spectral parameter $\omega$. We study also the case of irregular values of spectral parameter $\omega$. Our theorem proving methods are based on expanding the regular solution using a biorthogonal set of functions. The stability of the regular solution of the nonlocal problem with respect to the data is proved.

\section{REFERENCES}

1. Abdullaev O. Kh., Sadarangani K. Non-local problems with integral gluing condition for loaded mixed type equations involving the Caputo fractional derivative. Electron. J. Differential Equations, 2016. Vol. 2016. No. 164. P. 1-10. URL: https://ejde.math.txstate.edu

2. Application of Fractional Calculus in Physics. Hilfer R. (ed.) Singapore: World Scientific Publishing Company, 2000. 472 p. DOI: 10.1142/3779

3. Agarwal P., Berdyshev A., Karimov E. Solvability of a non-local problem with integral transmitting condition for mixed type equation with Caputo fractional derivative. Results Math., 2017. Vol. 71, P. 1235-1257. DOI: $10.1007 / \mathrm{s} 00025-016-0620-1$

4. Aziz S., Malik S.A. Identification of an unknown source term for a time fractional fourthorder parabolic equation. Electron. J. Differential Equations, 2016. Vol. 2016. No. 293. P. 1-20. URL: https://ejde.math.txstate.edu

5. Berdyshev A.S., Cabada A., Kadirkulov B. J. The Samarskii-Ionkin type problem for the fourth order parabolic equation with fractional differential operator. Comput. Math. Appl., 2011. Vol. 62. P. 38843893. DOI: $10.1016 /$ j.camwa.2011.09.038

6. Berdyshev A.S., Kadirkulov J.B. On a nonlocal problem for a fourth-order parabolic equation with the fractional Dzhrbashyan-Nersesyan operator. Differ. Equ., 2016. Vol. 52. No. 1. P. 122-127. DOI: 10.1134/S0012266116010109

7. Beshtokov M. Kh. Boundary value problems for the generalized modified moisture transfer equation and difference methods for their numerical implementation. Appl. Math. Phys., 2020. Vol. 52. No. 2. P. 128-138. DOI: 10.18413/2687-0959-2020-52-2-128-138 (in Russian)

8. Gel'fand I. M. Some questions of analysis and differential equations. Uspekhi Matem. Nauk., 1959. Vol. 14. No. 3. P. $3-19$ (in Russian).

9. Handbook of Fractional Calculus with Applications. Vols. 1-8. Tenreiro Machado J.A. (ed.). Berlin, Boston: Walter de Gruyter GmbH, 2019.

10. Hilfer R. Experimental evidence for fractional time evolution in glass forming materials. Chem. Phys., 2002. Vol. 284, No. 1-2. P. 399-408. DOI: 10.1016/S0301-0104(02)00670-5

11. Hilfer R. On fractional relaxation. Fractals, 2003. Vol. 11. No. Supp. 01. Part III: Scaling. P. 251-257. DOI: $10.1142 /$ S0218348X03001914

12. Hilfer R., Luchko Y., Tomovski Ž. Operational method for the solution of fractional differential equations with generalized Riemann-Liouville fractional derivatives. Fract. Calc. Appl. Anal., 2009. Vol. 12, No. 3. P. 299-318. URL: http://www.math.bas.bg/ fcaa/volume12/fcaa123

13. Kal'menov T. S., Sadybekov M. A. On a Frankl-type problem for a mixed parabolic-hyperbolic equation. Sib. Math. J., 2017. Vol. 58. No. 2. P. 227-231. DOI: 10.1134/S0037446617020057

14. Kerbal S., Kadirkulov B. J., Kirane M. Direct and inverse problems for a Samarskii-Ionkin type problem for a two dimensional fractional parabolic equation. Progr. Fract. Differ. Appl., 2018. Vol. 4. No. 3. P. 147-160. DOI: $10.18576 / \mathrm{pfda} / 040301$

15. Kim M.-Ha, Ri G.-Chol, O. H.-Chol. Operational method for solving multi-term fractional differential equations with the generalized fractional derivatives. Fract. Calc. Appl. Anal., 2014. Vol. 17. No. 1. P. 79-95. DOI: 10.2478/s13540-014-0156-6 
16. Kumar D., Baleanu D. Editorial: fractional calculus and its applications in physics. Front. Phys., 2019. Vol. 7. No. 6. P. 1-2. DOI: 10.3389/fphy.2019.00081

17. Malik S.A., Aziz S. An inverse source problem for a two parameter anomalous diffusion equation with nonlocal boundary conditions. Comput. Math. Appl., 2017. Vol. 73. No. 12. P. 2548-2560. DOI: 10.1016/j.camwa.2017.03.019

18. Nakhushev A. M. Zadachi so smeshcheniem dlya uravnenij $v$ chastnyh proizvodnyh [Problems with Displacement for Partial Differential Equations]. Moscow: Nauka, 2006. 287 p. (in Russian).

19. Patnaik S., Hollkamp J. P., Semperlotti F. Applications of variable-order fractional operators: a review. Proc. A., 2020. Vol. 476, No. 2234. P. 1-32. DOI: 10.1098/rspa.2019.0498

20. Sabitov K. B. Nonlocal problem for a parabolic-hyperbolic equation in a rectangular domain. Math. Notes, 2011. Vol. 89, No. 4. P. 562-567. DOI: 10.1134/S0001434611030278

21. Sabitov K. B. K teorii uravnenij smeshannogo tipa [On the Theory of Mixed Type Equations]. Moscow: Fizmatlit, 2014. 304 p. (in Russian).

22. Sabitov K. B., Sidorov S. N. On a nonlocal problem for a degenerating parabolic-hyperbolic equation. Differ. Equ., 2014. Vol. 50. No. 3. P. 352-361. DOI: 10.1134/S0012266114030094

23. Sandev T., Metzler R., Tomovski Ž. Fractional diffusion equation with a generalized Riemann-Liouville time fractional derivative. J. Phys. A. Math. Theor., 2011. Vol. 44, No. 25. Art. no. 255203.

24. Sandev T., Tomovski Ž. Fractional Equations and Models: Theory and Applications. Dev. Math., vol. 61. Cham, Switzerland: Springer Nature Switzerland AG, 2019. 345 p. DOI: 10.1007/978-3-030-29614-8

25. Saxena R. K., Garra R., Orsingher E. Analytical solution of space-time fractional telegraph-type equations involving Hilfer and Hadamard derivatives. Integral Transforms Spec. Funct., 2015. Vol. 27, No. 1. P. 30-42. DOI: $10.1080 / 10652469.2015 .1092142$

26. Sun H., Chang A., Zhang Y., Chen W. A review on variable-order fractional differential equations: mathematical foundations, physical models, numerical methods and applications. Fract. Calc. Appl. Anal., 2019. Vol. 22, No. 1 P. 27-59. DOI: 10.1515/fca-2019-0003

27. Terlyga O., Bellout H., Bloom F. A hyperbolic-parabolic system arising in pulse combustion: existence of solutions for the linearized problem. Electron. J. Differential Equations, 2013. Vol. 2013. No. 46. P. 1-42. URL: https://ejde.math.txstate.edu

28. Uflyand Ya. S. K voprosu o rasprostranenii kolebanij v sostavnyh elektricheskih liniyah [On oscillation propagation in compound electric lines]. Inzhener.-Phis. Zhurn. [J. of Engineering Physics and Thermophysics], 1964. Vol. 7. No. 1. P. 89-92 (in Russian).

29. Yuldashev T.K. Nonlocal problem for a mixed type differential equation in rectangular domain. Proc. Yerevan State Univ. Phys. Math. Sci., 2016. No. 3. P. 70-78.URL: http://mi.mathnet.ru/uzeru278

30. Yuldashev T.K. Solvability of a boundary value problem for a differential equation of the Boussinesq type. Differ. equ., 2018. Vol. 54. No. 10. P. 1384-1393. DOI: 10.1134/S0012266118100099

31. Yuldashev T.K. A coefficient determination in nonlocal problem for Boussinesq type integro-differential equation with degenerate kernel. Vladikavkaz. Mat. Zh., 2019. Vol. 21. No. 2. P. 67-84 (in Russian). DOI: $10.23671 /$ VNC.2019.2.32118

32. Yuldashev T.K. On a boundary-value problem for Boussinesq type nonlinear integro-differential equation with reflecting argument. Lobachevskii J. Math., 2020. Vol. 41. No. 1. P. 111-123. DOI: $10.1134 /$ S1995080220010151

33. Yuldashev T. K. Nonlocal inverse problem for a pseudohyperbolic-pseudoelliptic type integro-differential equations. Axioms, 2020. Vol. 9. No. 2. Art. no. 45. P. 1-21. DOI: 10.3390/axioms9020045 